\title{
Topological analysis of tapped granular media using persistent homology
}

\author{
S. Ardanza-Trevijano, ${ }^{1, *}$ Iker Zuriguel,,${ }^{1, \dagger}$ Roberto Arévalo, ${ }^{2}$ and Diego Maza ${ }^{1}$ \\ ${ }^{1}$ Departamento de Física, Facultad de Ciencias, Universidad de Navarra, 31080 Pamplona, Spain \\ ${ }^{2}$ CNR-SPIN, Dipartimento di Scienze Fisiche, Università di Napoli Federico II, I-80126 Napoli, Italy
}

(Received 15 October 2013; published 23 May 2014)

\begin{abstract}
We use the first Betti number of a complex to analyze the morphological structure of granular samples in mechanical equilibrium. We investigate two-dimensional granular packings after a tapping process by means of both simulations and experiments. States with equal packing fraction obtained with different tapping intensities are distinguished after the introduction of a filtration parameter which determines the particles (nodes in the network) that are joined by an edge. This is accomplished by just using the position of the particles obtained experimentally and no other information about the possible contacts, or magnitude of forces.
\end{abstract}

DOI: 10.1103/PhysRevE.89.052212

PACS number(s): 45.70.Cc, 64.60.aq

\section{INTRODUCTION}

Dense granular media can be considered as an archetypical example of a complex system where even the simplest case of a monodisperse disk packing can display nontrivial features, like arching or jamming. In fact, the geometrical confinement imposed by the boundaries to the granular sample implies mechanical restrictions that must be equilibrated by the interparticle contact forces. It has not been until recently that the structural complexity of this kind of system has been described formally. For a review on this problem, see [1]. Edwards and co-workers [2] proposed a statistical mechanical theory of granular materials where energy and volume were replaced by volume and compactivity respectively. Thus, systems with the same density and number of particles, and reachable from one another, would be equivalent. The application of this idea to real situations evidenced that the approach was unable to fully describe the states reached by a granular ensemble, and a new variable, the angoricity, was introduced [3]. Both magnitudes are inherently related with the structural complexity of the packing and both are, indeed, necessary to describe unequivocally any granular solid [4]. Importantly, this description seems to be strongly dependent on the tessellation of the space which is implemented by the introduction of elementary volume elements. Those are necessary to construct the partition function that defines the ensemble of volume states. In consequence, it is important to introduce geometrical or topological tools to describe the spatial arrangement of the elements that determine the equilibrium state of a static granular ensemble.

Under these circumstances, it seems natural and appealing to consider a granular system as a graph where contacts between particles are edges, and the corresponding particles are nodes. Defining such a network can be useful since it can be analyzed using the machinery of topology and modern complex networks theory [5]. The contact network has been used to study a large variety of global properties of disordered media [6-8]. This approach can be also used to analyze the evolution of granular media in dynamic situations [9-11]. In [12] the topological properties of the network were related to the

\footnotetext{
*sardanza@unav.es

†iker@unav.es
}

process of strain localization, which leads to shear banding and material failure. Related with failure is the process of buckling of force chains studied in [13], where the importance of the presence of loops of contacts in the network was revealed. These loops were also proven to be crucial in the stability of other granular systems [14,15]. A similar approach-where the loops were called mesodomains-allowed us to analyze the loop orientation within the sample and relate it to the stress transmission [16,17].

Additionally to the contact network (the graph of all contacts), the force network can be also analyzed with the same methods. The normalized contact force $f=F /\langle F\rangle$ (where $F$ is the force present in a contact and $\langle F\rangle$ is the sample average) can be used to define as the edge any contact bearing a force $f$ larger than some threshold value $f^{*}$ that can be tuned in the range $\left[0, f_{\max }\right]$. Thus, for $f^{*}=0$ one recovers the contact network, while for larger values one obtains progressively diluted graphs. The analysis of the topology of the force networks has been shown very fruitful $[18,19]$ in a static granular packing. The process of jamming in the light of the topology of force networks was studied in [20,21]. Again, the role of loops in the network was proven to be relevant at the transition point, with third-order loops behaving as an order parameter. Related to jamming is the question of isostaticity, which was analyzed using the force network by Walker et al. [22].

A promising perspective has been recently introduced by Kondic and co-workers who analyzed the force network using topological invariants. In [23] the zeroth Betti number $\beta_{0}$, which measures the number of connected components (clusters), is used to study compressed granular samples. $\beta_{0}$ is shown to be useful characterizing force networks obtained with varying density, friction, and polydispersity of the grains. The zeroth Betti number is also used in [24] to analyze the role of interparticle friction in impact dynamics. Carlsson et al. [25] used the zeroth and first Betti numbers to analyze a 2D system with small number of particles. Interestingly, they showed that critical points (where the topology can change) correspond to configurations in mechanical equilibrium.

Considering all these works, it follows that studying contact and force networks offers a fruitful pathway for the understanding of static and dynamic properties of granular media. It is not completely clear, however, if these tools provide more (or better) information than other traditional tools used 
to explore the relation between geometry and topology [26]. This question was addressed in [27] where the topology of $2 \mathrm{D}$ granular samples in mechanical equilibrium, in the sense of Edwards' theory, was studied. It was already known [28] that samples with the same density and number of particles may not be in the same state of equilibrium since the average force moment tensor can be different. In [27] it was shown that the topology of the contact network (without information on the forces) was enough to distinguish these mechanically different states. Interestingly, traditional measurements based on particles' positions-like the pair correlation function, the bond order parameter and the Voronoi tessellation-were shown to be less sensitive to capture such differences among different states with the same packing fraction. In the same line is the recent work of Kramar et al. [29] who have used persistent homology to study the evolution of the force network in compressed granular materials. Their approach is able to uncover the distinctive behavior displayed by different systems and, moreover, it is shown to be richer in information than the pair-correlation function, the bond orientational order parameter, and the distribution function of the forces.

Most of the works mentioned here are theoretical or consist of numerical simulations where one has all the information necessary to construct the contact and force networks. In the last years, there has been also an important experimental effort aimed at identifying particle contacts (or forces) which are then used to construct a network [30,31]. However, under experimental conditions it is always difficult to establish with certainty if there is contact between adjacent particles. It is then desirable to devise a robust method to study the contact network when contacts cannot be exactly determined. In the present work we aim at precisely this goal using persistent homology.

The system on which we implement persistent homology is a granular bed subjected to tapping, which has the appeal of being a proving ground for the statistical theory of granular media in mechanical equilibrium. Thus, it has also been widely studied experimentally [32-36] and by means of simulations $[28,37,38]$. In $[28,38]$ it was shown that the packing fraction $\phi$ of the bed is not a monotonous function of the tapping intensity $\Gamma$. This raises the question of whether states with the same density are equivalent or not, in the sense of the statistical mechanical theory. A negative answer to this question was given in [38] analyzing the force moment tensor of the system. As mentioned before, the same result can be obtained using the contact network. In the present work we experimentally show that even when contacts among particles are not known, persistent homology allows us to distinguish between states with the same density but in different mechanical equilibrium.

\section{EXPERIMENTS}

A quasi-2D Plexiglass cell (width $28 \mathrm{~mm}$, height $150 \mathrm{~mm}$ ) was used to study the dependence of the packing on the intensity of shaking. The cell was filled with 600 alumina oxide spheres of diameter $d=1.00 \mathrm{~mm}$. The side wall separation was $10 \%$ larger than the bead diameter in order to minimize the particle-wall friction and prevent arching in the transversal direction. The system was tapped with an electromagnetic
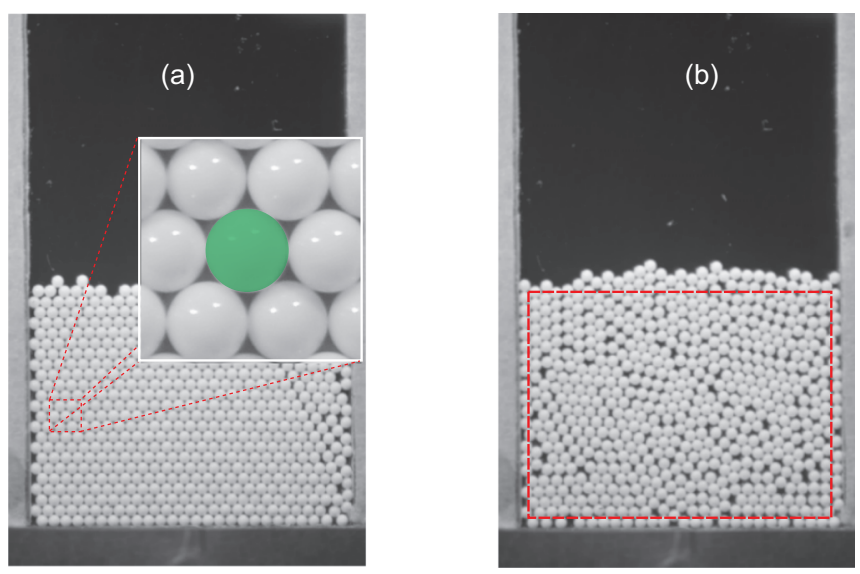

FIG. 1. (Color online) Experimental pictures of two packings obtained with (a) $\Gamma=3.5$ and $\Gamma=14.9$. In (a) a closeup of the image is shown evidencing the light diffraction. The (green) disk indicates the particle recognition as performed experimentally. In (b) the region where the packing fraction is measured is displayed by a (red) dashed line.

shaker that provides a sine shaped pulse with a frequency $(v)$ and an amplitude $(A)$. The frequency was kept constant at $(v=30 \mathrm{~Hz})$ and the amplitude was systematically modified in order to vary the tapping intensity $\Gamma=\frac{A(2 \pi \nu)^{2}}{g}$. The latter was measured with a piezoelectric accelerometer attached to the base of the cell.

High-resolution digital images of the packings were taken after each tap (Fig. 1). The packing fraction was calculated by considering each grain as a disk of the corresponding effective diameter [see the shadow particle in the inset of Fig. 1(a)] and then calculating the percentage of the area covered by the disks in the rectangular area displayed in Fig. 1(b). Although the wall separation could induce an overlap between some beds in the front view, a simple calculation shows that its value represents only $0.6 \%$ of the particle diameter. Nevertheless, as evidenced in the inset of Fig. 1(a), the main source of error is the diffraction of light at the border of particles that makes it difficult to determine the position of the centers with subpixel resolution. We estimate that in standard experimental conditions, the error in the calculation of the particle center position is around $2 \%$ of the particle diameter. In order to determine the average packing fraction of a state, we average over the last 200 packings for each tapping amplitude, after reaching the stationary state. This is determined when its fluctuations - measured by its standard deviation-are stationary. More details of this analysis can be found in [38].

Using this procedure we obtain the curve of density versus tapping intensity shown in Fig. 2. For low values of $\Gamma$ the bed remains very compact, in a quasicrystallized state. Note that the asymptotic value of the mean packing fraction for small $\Gamma$ is larger than the theoretical limit $\frac{1}{6} \pi \sqrt{3} \approx 0.907$ due to the combined effects of the 3D bead superposition and the errors in the particle's position estimation. When $\Gamma$ is increased, the mean packing fraction decreases until it reaches a minimum at a certain $\Gamma_{\min }$. After this value, the tendency is reversed and the sample becomes compacted as $\Gamma$ is increased. 


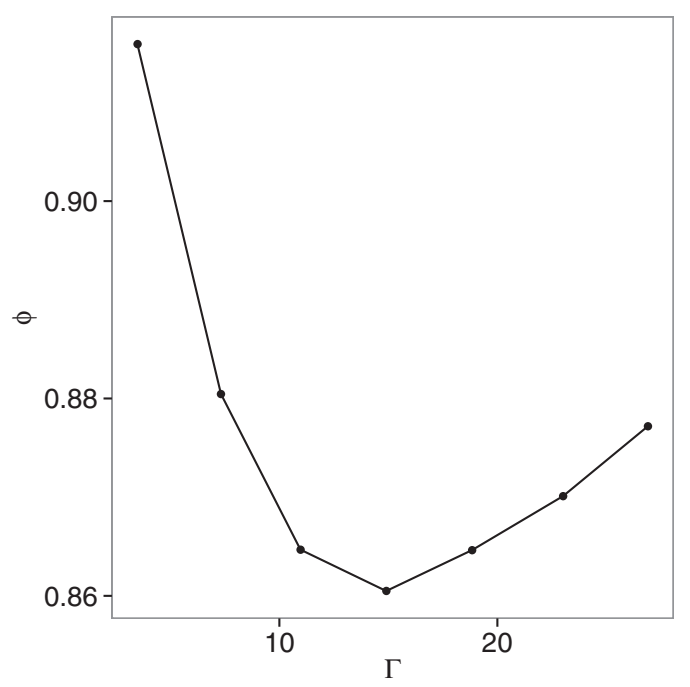

FIG. 2. Experimental results of the mean packing fraction $\phi$ of the steady states as a function of the tap intensity $\Gamma$.

The nonmonotonicity of this curve implies that steady states with the same packing fraction can be reached using very different values of tapping intensity. These states of equal $\phi$ have been demonstrated to display completely different stress properties [38], being also distinguishable by measuring the number of polygons of $3,4,5,6 \ldots$ sides, obtained from the contact network [27]. This method, however, is dramatically dependent on the exact determination of the particle-particle contacts, which is in general impossible in any experimental situation. In what follows, we show how persistent homology is implemented to distinguish among states with the same packing fraction using the experimental data and a filtration parameter which is used to build a collection of granular networks.

\section{PERSISTENT HOMOLOGY}

Persistent homology is a tool that provides topological information of an object examined at different resolutions. We will give an ad hoc description in the following paragraph and recommend the interested reader the sources [39-41] for a more detailed and broad description. Since our data are 2D we will restrict all the relevant constructions to two dimensions.

Our data are the position of the centers of the particles, i.e., a set of points in the plane. The natural way to build a contact network is to consider the graph that has as vertices the mentioned set of points, and add an edge between a pair of vertices $p_{i}$, $p_{j}$, if the Euclidian distance between them is less than or equal to the diameter $d$ of the particles $d\left(p_{i}, p_{j}\right) \leqslant d$. However, this construction will miss some existing contacts and add some nonexisting ones due to experimental uncertainties. To deal with this problem we construct a parametrized collection of graphs where the vertices are the particle's centers, and the edges in each graph are added whenever the distance between two vertices is smaller than a parameter $\delta \geqslant 0$. In Fig. 3 we evidence that the network obtained using $\delta=d$ is quite unrealistic due to the lack of precision in the determination of the particles' centers. As $\delta$ increases, more contacts appear in the complex. Obviously, some of them are spurious as
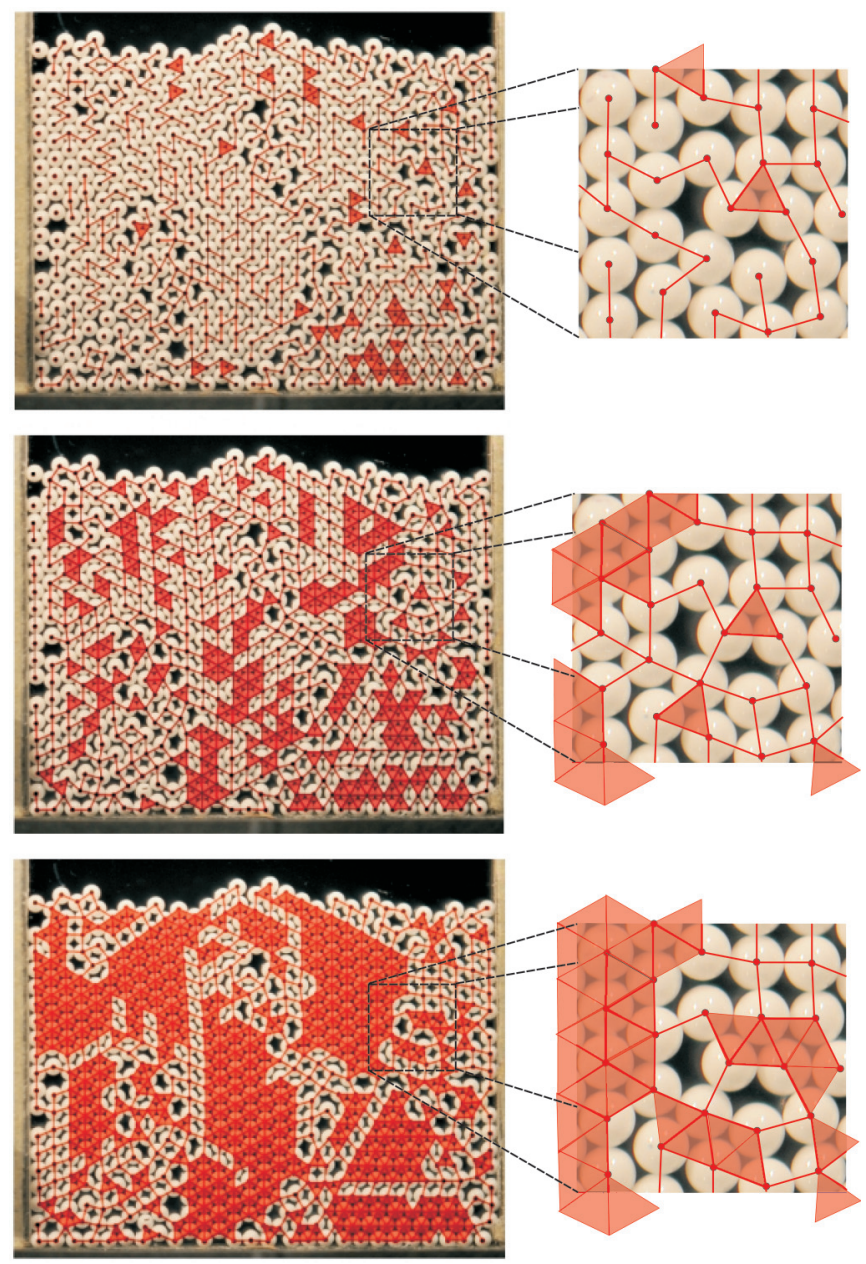

FIG. 3. (Color online) Three examples of the Vietoris-Rips complex (clique complex of the graph) obtained from the data given by the centers of particles of a sample obtained with $\Gamma=11$ which has an associated value of packing fraction $\phi=0.864$. Each line corresponds to a different value of the filtration parameter $\delta$. Top: $\delta=d$ the exact diameter of the particle. Center: $\delta=1.01 d$. Bottom: $\delta=1.05 d$. A magnified region is shown evidencing the effect of increasing the filtration parameter in the Vietoris-Rips complex: some quadrilaterals in the $\delta=1.01 \mathrm{~d}$ picture are converted in triangles in the $\delta=1.05 d$ picture. As explained in the text, the creation of a triangle implies an augment of $\beta_{1}$ in the graph, and (in most cases) a decrease of $\beta_{1}$ in the associated clique complex.

they are not real contacts, specially for the bottom picture where $\delta=1.05 \mathrm{~d}$. These three pictures evidence the difficulty of properly defining a network of contacts from experimental data.

Three particles that are in contact with each other form a triangular structure which can be viewed as a "local perfect packing." In order to keep track of these, we build a second structure associated with each one of the previously described graphs. If three nodes (particles) have all pairwise connections, i.e., edges between them form a triangle, we add a $2 \mathrm{D}$ cell covering the triangle. We thus obtain a sort of "tesselation with holes" (see Fig. 3), in which the "holes" are the polygons formed by closed loops in the graph that are not triangles. This structure is a $2 \mathrm{D}$-simplicial complex that is usually called the 
2D-clique complex of the graph, and also the 2D-Vietoris-Rips complex of the dataset for the given filtration parameter.

Once we have a simplicial complex we can calculate its Betti numbers which are non-negative integers, one for each dimension. Since our complexes live in the Euclidean plane, we are only interested in zero-dimensional and onedimensional Betti numbers. The zeroth Betti number $\left(\beta_{0}\right)$ of a complex is the number of connected components, and the first Betti number $\left(\beta_{1}\right)$ counts the number of $1 \mathrm{D}$ holes (the network polygons) in our complex. We will calculate the first Betti number $\left(\beta_{1}\right)$ of both the graph and the clique complex. As mentioned above, $\beta_{1}$ in the graph accounts for the total number of $1 \mathrm{D}$ holes, i.e., the number of polygons given by edges connecting data points in the graph. In the clique complex, $\beta_{1}$ provides the number of uncovered polygons, i.e., polygons that are not triangles. Due to the presence of gravity, we expect to have a single connected component in most cases $\left(\beta_{0}=1\right)$, and thus we focus our study only in $\beta_{1}$. In this article, the homology calculations have been performed with JAVAPLEX [42], developed by the group of Applied and Computational Algebraic Topology of Stanford University. These calculations were subsequently corroborated using PERSEUS $[43,44]$, developed by V. Nanda.

\section{TOPOLOGY UNCOVERED BY $\beta_{1}$}

The goal now is testing if the average first Betti number, combined with the introduction of a filtration parameter, can be used to unveil the characteristics of the different packings. The effect of increasing the filtration parameter above the diameter of the particles in the $\beta_{1}$ of the graph is that the development of new connections necessarily leads to the apparition of polygons and hence, to the increase of $\beta_{1}$. In the clique complex, however, new connections may lead to the creation of polygons, but also to the covering of a triangle (and hence to a reduction of $\beta_{1}$ ) as evidenced in Fig. 3. We study filtration parameters in the range $d \leqslant \delta \leqslant 1.1 d$ where $d$ denotes the diameter of the particles.

The evolution of $\beta_{1}$ versus the tapping intensity is presented in the left column of Fig. 4 for both, the graph (top) and the clique (bottom). In both cases, the curves obtained for $\delta=d$ display considerably lower values than the other ones. This correlates with the pictures shown in Fig. 3 and the unrealistic type of network obtained for this value of the filtration parameter. Interestingly, apart from the quantitative disparity, the curves obtained for $\delta=d$ display qualitative differences from the ones using larger values of $\delta$. For the case of the graph, the values of $\beta_{1}$ are rather homogeneous for $\delta=d$ whereas a nonmonotonous behavior can be observed for $\delta \geqslant 1.01 d$. This nonmonotonicity can be understood if we consider the packing fraction dependence on $\Gamma$ shown in Fig. 2. Indeed, this behavior is in good agreement with the results reported in [27] where the total number of polygons (dominated by the number of triangles) was shown to change with $\Gamma$ in the same way that the packing fraction does. The curves reported for $\beta_{1}$ of the clique (where the triangles are not considered) show that the packing structure is dominated by the presence of triangular loops. First, the values of $\beta_{1}$ of the clique are (at least) half the ones obtained for the graph. In addition, the presence of the maximum of $\beta_{1}$ for the value
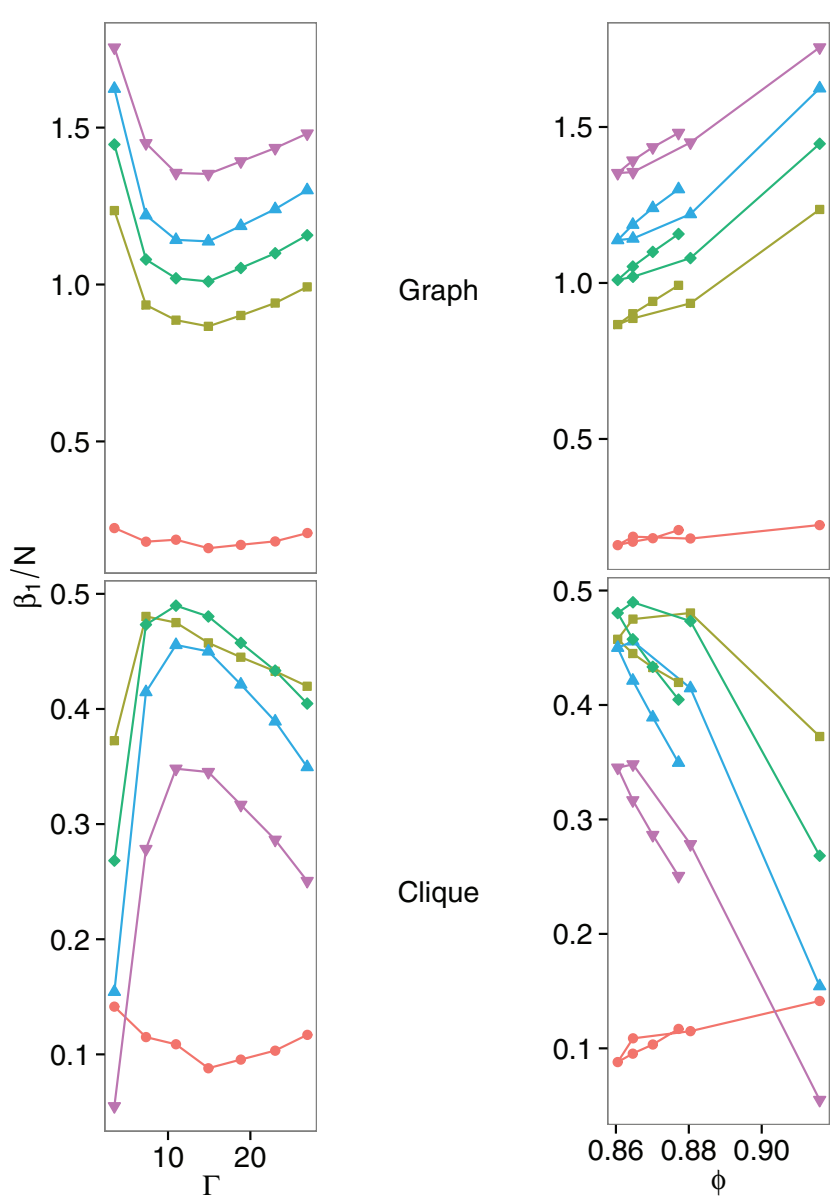

$\rightarrow \delta=1 \mathrm{~d} \rightarrow \delta=1.01 \mathrm{~d} \rightarrow \delta=1.02 \mathrm{~d} \rightarrow \delta=1.04 \mathrm{~d} \rightarrow \delta=1.12 \mathrm{~d}$

FIG. 4. (Color online) Experimental results of the mean first Betti number $\left(\beta_{1}\right)$ normalized by the number of particles for different values of the filtration parameter $\delta$ as indicated in the legend. In the left column, $\beta_{1}$ is presented vs the tap intensity $\Gamma$. In the right column $\beta_{1}$ is presented vs the packing fraction of the sample $\phi$. At the top, results obtained from the graph and at the bottom, results obtained from the clique. In all cases, the $95 \%$ confidence intervals for the mean of the normalized Betti numbers are of the size of the data points.

of $\Gamma$ at which we obtain the smaller packing fraction indicates that for these states the number of polygons with four or more edges are maximized and the number of triangles is minimized. On the contrary, as we increase or decrease $\Gamma$ and the packing fraction is increased, the number of triangles augments with the consequent reduction of the number of polygons with four or more edges.

All these results can be better understood if we plot $\beta_{1}$ versus $\phi$ (right column of Fig. 4). The graph shows an increase of $\beta_{1}$ with $\phi$ independently on the value of $\delta$ confirming the correlation of these two parameters. On the contrary, the clique displays a decrease of $\beta_{1}$ with $\phi$, confirming that the behavior observed in the graph is dominated by the presence of triangular structures as explained above. Even more interestingly, the $\beta_{1}$ versus $\phi$ curves reveal that, for states 


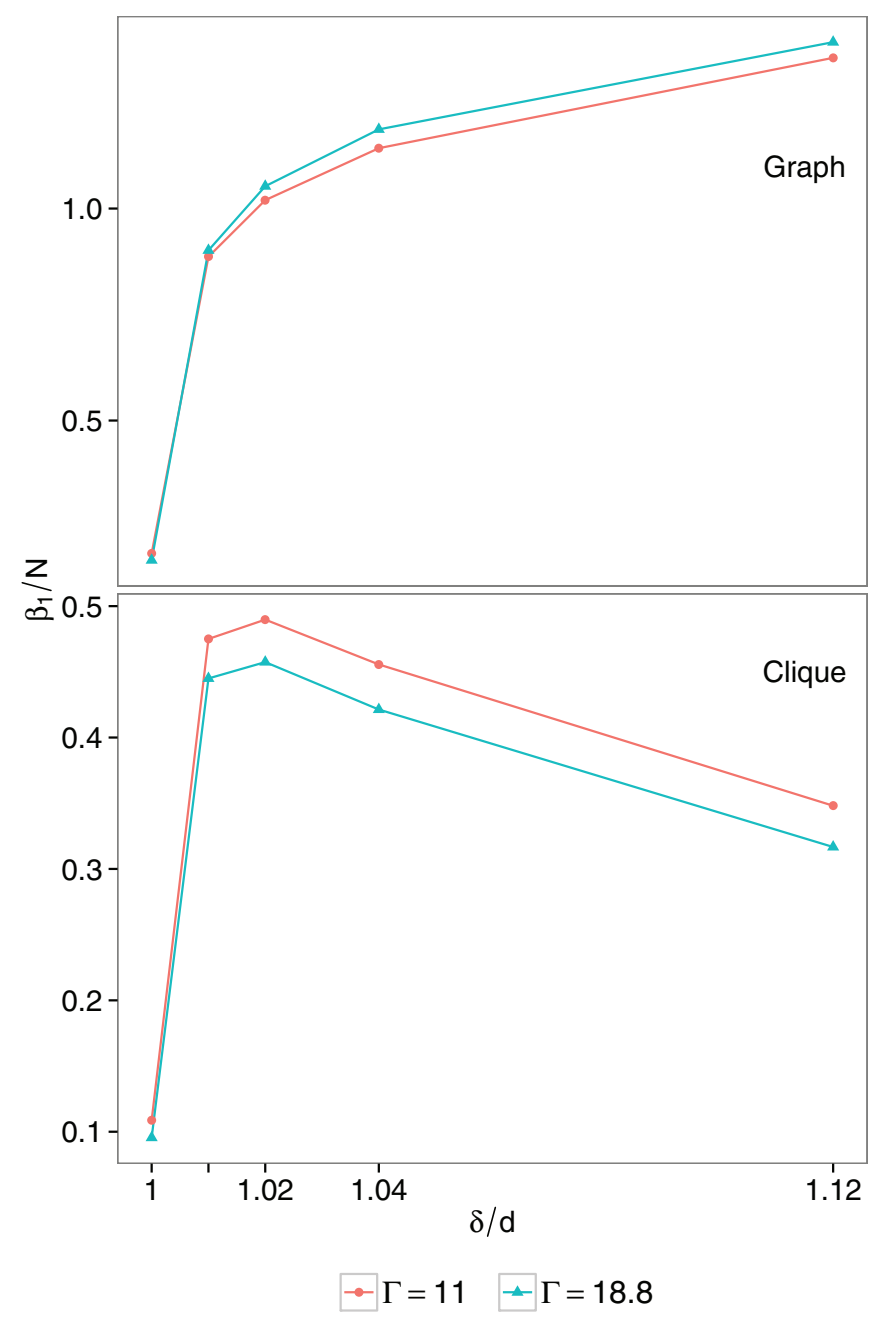

FIG. 5. (Color online) Mean first Betti number vs $\delta$ for states with the same average packing fraction $(\phi \approx 0.864)$ obtained with different tap intensities. As indicated in the legend, circles (triangles) are used for the state reached with $\Gamma=11(\Gamma=18.8)$. At the top, results obtained from the graph and at the bottom, results obtained from the clique. The $95 \%$ confidence intervals for the mean of the normalized Betti numbers are of the size of the data points.

with the same value of $\phi$ but obtained with different excitation intensities, the $\beta_{1}$ values are different. This is evidenced by the presence of two branches in the plots. The branch corresponding to high $\Gamma$ displays higher $\beta_{1}$ values for the graph and lower $\beta_{1}$ values for the clique. This suggests that, even if two states share the same packing fraction, those obtained with the higher excitations can be identified by the presence of a larger number of triangular structures in the network.

In order to check this idea, in Fig. 5 we compare the experimental values of $\beta_{1}$ for two states with the same packing fraction but obtained at different tap intensities, i.e., $\Gamma=11$ and $\Gamma=18.8$ for the left and right sides of $\Gamma_{\min }$. In the graph, the $\beta_{1}$ values obtained for the highest $\Gamma$ are systematically above those obtained for the lowest $\Gamma$. This trend is reversed for the clique, where the $\beta_{1}$ values obtained for the highest $\Gamma$ are systematically below those obtained for the lowest $\Gamma$. This behavior is in perfect agreement with the fact that the number of triangles developed in the network is more important for the higher value of $\Gamma$.

Finally, let us stress that from the results shown in Figs. 4 and 5, it can be concluded that a good election of the filtration parameter is crucial in order to differentiate among states with the same packing fraction. Indeed, the network constructed in the traditional way (considering as links those nodes at a distance equal to or smaller than the particle diameter) is shown to be the worst election to differentiate among states. On the contrary, a filtration parameter of $\delta=1.01 d$ or $\delta=1.02 d$ seems to be the most convenient for the experiments displayed in this work. Noticeably, these values of the filtration parameter are of the same order as the indetermination of the particle's positions explained above. In the next section we show that, effectively, there is a relationship among these two magnitudes. In a recent paper [45] a slightly larger number (8\% of particle diameter) has been found to be a good election for contact threshold.

At this point, we have shown that the first Betti number of the graph and the clique (the Vietoris-Rips complex) can be satisfactorily used to classify granular packings. In the next section we show that our conclusions are robust against the inherent noise of experimental measurements. To this end, we use numerical simulations and artificially introduce different degrees of noise in the positions of the particles.

\section{INFLUENCE OF PARTICLE'S POSITION INDETERMINACIES}

In order to validate our experimental results and check the role that particle position indetermination has on the $\beta_{1}$ values obtained for different filtration parameters, we use data of previous soft-particle molecular dynamics in two dimensions obtained for a very similar geometry. The details of the simulations can be found elsewhere [46] and are summarized in the Appendix. Here we discuss the results.

The main source of errors in our experimental results comes from the uncertainties in the determination of the particle position; we simulate this process by adding controlled noise to the numerical particles' positions which can be obtained with $10^{-8} d$ precision. Therefore, we created sets of noisy data with a well defined protocol: defining as noise control parameter the value of $\alpha$ in $[0,0.1]$, we moved each center to a point at a random distance sampled from a uniform distribution in $[0, \alpha d]$ where $d$ is the diameter of the particles, and a random direction sampled from a uniform distribution in $[0,2 \pi]$.

In Fig. 6 results of mean $\beta_{1}$ (normalized by the number of particles) are presented versus $\Gamma$ for different values of the filtration parameter $\delta$ (as indicated in the legend) and different levels of noise (increasing from left to right panels). Looking at the results of the graph obtained for $\delta=1 d$ without noise (circles in the top left panel), we notice the same qualitative behavior as the one observed in [27] for the total number of polygons in the contact network. In the same panel, we observe that increasing the value of $\delta$ leads to an augment of the values of $\beta_{1}$ (the number of polygons increases) preserving the shape of the curves. Furthermore, when noise is added to the data, the curves $\beta_{1}$ versus $\Gamma$ in the graph (top figures of Fig. 6) show an important downward displacement of some of the curves but the global trends are maintained. Adding $1 \%$ noise 


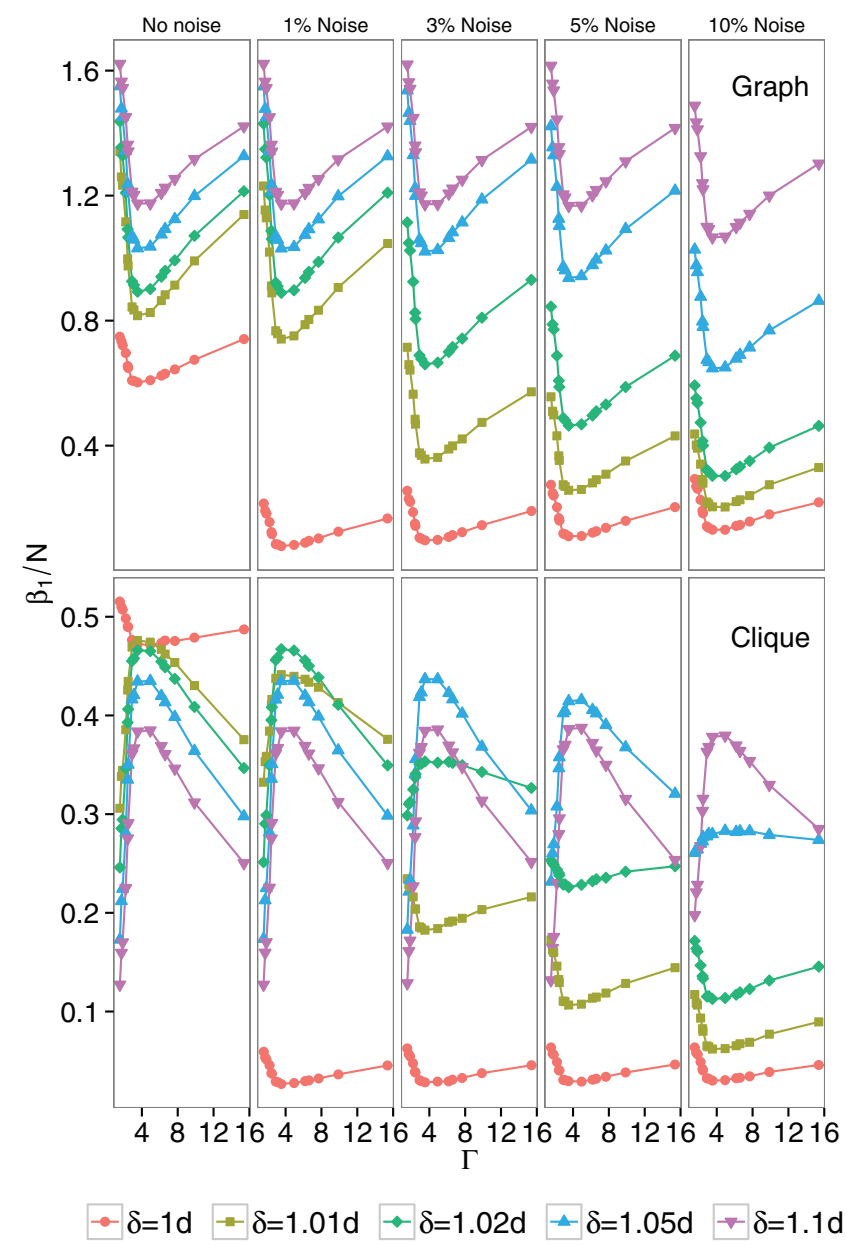

FIG. 6. (Color online) Simulation results of the mean first Betti number $\left(\beta_{1}\right)$ normalized by the number of particles as a function of the tap intensity $(\Gamma)$ for different values of the filtration parameter $\delta$ and different levels of noise in the data. At the top, results obtained from the graph and at the bottom, results obtained from the clique. The $95 \%$ confidence intervals for the mean of the normalized Betti numbers are of the size of the data points.

strongly (slightly) affects $\delta=1.00 d(\delta=1.01 d)$ curves and has no apparent effect on curves obtained for higher values of $\delta$. Adding 3\% noise strongly affects $\delta=1.00 \mathrm{~d}, \delta=1.01 \mathrm{~d}$, and $\delta=1.02 d$ curves; adding $5 \%$ noise strongly affects curves with $\delta<1.05 d$, and weakly affects $\delta=1.05 d$.

A rather different behavior is obtained when displaying the values of $\beta_{1}$ for the clique (bottom panels in Fig. 6). We will start explaining the case without noise (bottom left panel). Although the trend displayed for $\delta=1 d$ is similar to that obtained in the graph, a small increase of $\delta$ leads to a change of the curve trend: the minimum is transformed into a maximum. Considering that the only difference between the graph and the clique is that the latter does not account for triangles, the comparison of the correspondent curves provides interesting information. Focusing on the case of $\delta=1.01 d$, the fact that the $\beta_{1}$ of the clique increases with $\Gamma$ and then, after $\Gamma_{\min }$, decreases again implies that the number of polygons-without considering triangles-is maximum in $\Gamma_{\min }$. At this same point, the total number of polygons ( $\beta_{1}$ of the graph) was proven to be minimum. This reflects that, as in the experiments, the increase in $\beta_{1}$ of the graph obtained when we move apart from $\Gamma_{\text {min }}$ is due to an augmentation in the number of triangles and a reduction in the number of the other polygons. In the curves obtained increasing $\delta$ above $1.01 d$ (which leads to increasing values of $\beta_{1}$ in the graph) is observed a reduction of $\beta_{1}$ in the clique without alteration of the curve trend. This evidences that most of the polygons that are built in the graph when increasing $\delta$ are, indeed, triangles.

In the clique curves, the effect of adding noise is also notably different from that observed in the graph. If the value of $\delta$ is higher than the level of noise, the curves show a maximum and the values of $\beta_{1}$ are reduced as $\delta$ increases. On the contrary, if $\delta$ is smaller than the noise level, the curves that originally displayed a maximum invert their shape and show a minimum - revealing a trend similar to the one observed for the case without noise and $\delta=1 d$. This effect can be explained as follows. First, it should be recalled that for the case without noise, increasing $\delta$ leads to the development of a maximum in the clique curves as a consequence of the increase in the number of triangles. Considering this, it seems reasonable that adding a given amount of noise destroys some of the triangles creating polygons of any kind. The only way to compensate the addition of noise (and preserve the triangular structure in the network) is applying a sufficiently high filtration parameter.

In Fig. 7 we represent $\beta_{1}$ (for different values of noise and filtration parameter) with respect to the packing fraction $\phi$. As in the experiments all the $\beta_{1}$ curves present two well defined branches; the shorter one is for high $\Gamma$ and the longer one is for low $\Gamma$. These branches are more or less separated from each other depending on the values of noise and filtration parameter. Focusing first on the results of the graph without noise for $\delta=1.00 \mathrm{~d}$, we observe that $\beta_{1}$ increases with $\phi$, but this increment is more pronounced for the short branch (higher values of $\Gamma$ ). Comparing these results with the analog of the clique, where the two branches are indistinguishable, we can conclude that the differences among the two branches are predominantly caused by the development of triangles (which are more abundant for high values of $\Gamma$ ). This result agrees with the experimental outcomes shown above and the topological analysis carried out in [27].

The effect of increasing $\delta$ in the graph obtained without noise is just an augmentation of the values of $\beta_{1}$ without changing the shape of the curves. Nevertheless, an exceedingly high filtration parameter like $\delta=1.10 d$ seems to provoke a reduction in the separation between the two branches (down triangles in the top left graph of Fig. 7). The introduction of noise induces a decrease of the $\beta_{1}$ values of the graphs that mainly affects the curves obtained with a filtration parameter smaller than the level of noise.

In the data obtained from the clique, the effect of adding noise and changing the filtration parameter leads, in some circumstances, to an inversion of the tendency of the curves. Focusing first in the the case without noise, if $\delta>1.00 d$, $\beta_{1}$ decreases with $\phi$ in contrast to the case of $\delta=1.00 \mathrm{~d}$. The origin of this change (which was already explained when describing the results displayed in Fig. 6) is based in the development of triangles for high values of $\phi$. The introduction of noise in the system leads to the transition from ascendent to descendent curves appearing for larger values 


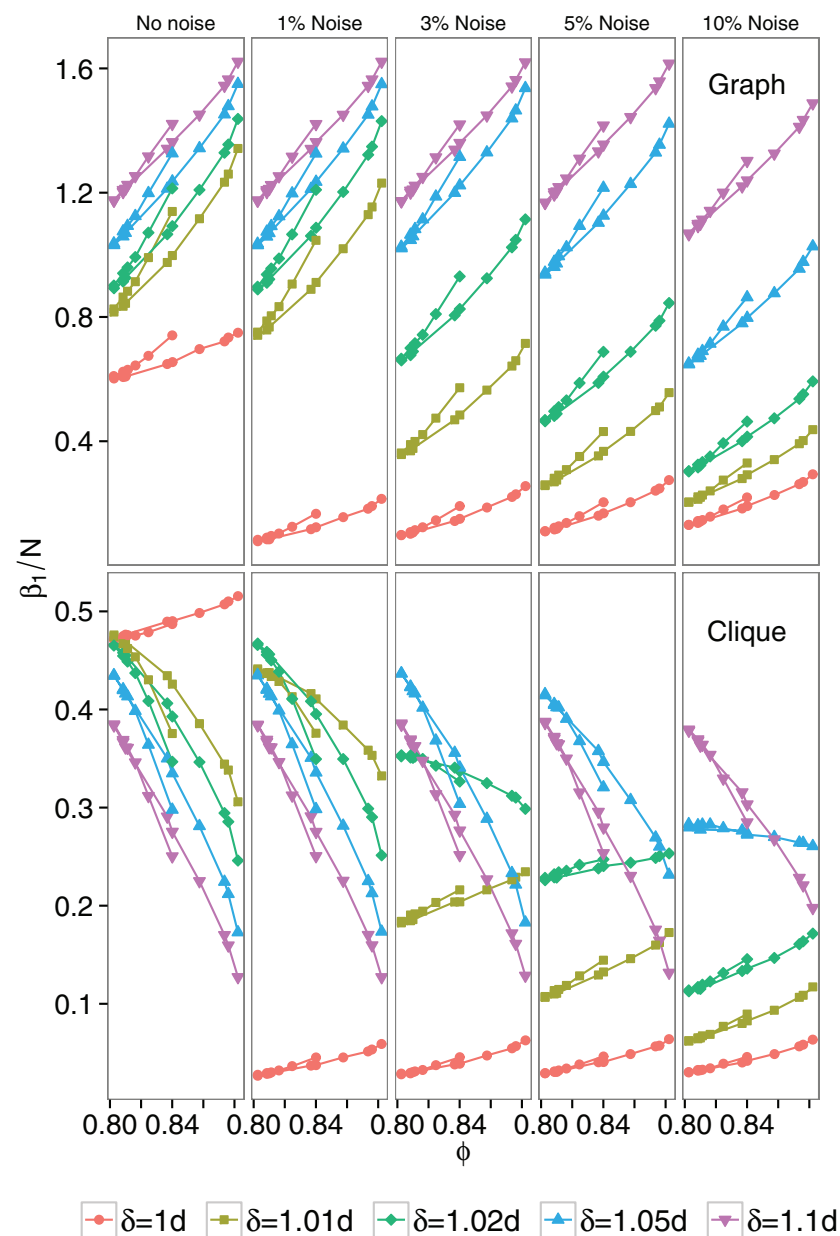

FIG. 7. (Color online) Simulation results of the mean first Betti number $\left(\beta_{1}\right)$ normalized by the number of particles as a function of the packing fraction $(\phi)$ for different values of the filtration parameter $\delta$ and different levels of noise in the data. At the top, results obtained from the graph and at the bottom, results obtained from the clique. The 95\% confidence intervals for the mean of the normalized Betti numbers are of the size of the data points.

of $\delta$. More interestingly, it seems that given a value of noise, the differences among the two branches in the clique networks are maximized for a filtration parameter higher than or similar to the level of noise.

In order to check this idea, we compare the outcomes of the $\beta_{1}$ for two states that, being obtained with very different tap intensities, display the same packing fraction $(\phi \approx 0.84)$. In the numerical simulations this occurs, for example, for the states developed for $\Gamma=2.4$ and $\Gamma=15.4$ whose $\beta_{1}$ values for different noise and filtration parameters are presented in Fig. 8. Clearly, the results obtained for the graph (top figures) reveal differences, the values of $\beta_{1}$ being systematically higher for the highest tapping intensity. The differences become more or less important depending on the noise and the filtration parameter. For the data without additional noise, it seems that the outcomes of $\beta_{1}$ are already different for $\delta=1 d$. The differences are magnified for larger values of $\delta$ and seem to become smaller again for $\delta=1.1 d$. Similar trends are observed when noise is added in the

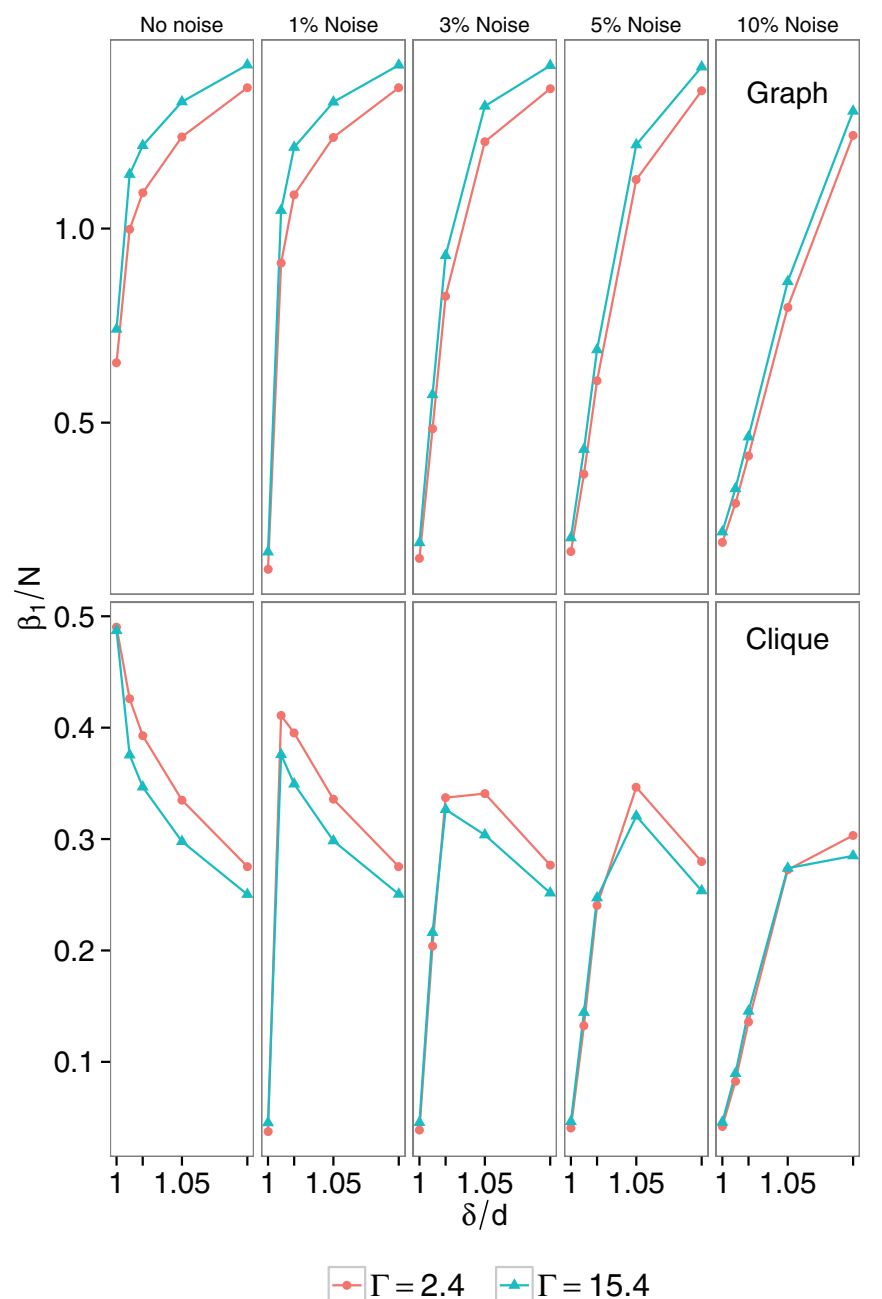

FIG. 8. (Color online) Comparison of the mean first Betti number normalized by the number of particles for states with the same packing fraction obtained with different tap intensities $(\Gamma=2.4$ and $\Gamma=$ 15.4). Results obtained from simulations are presented vs the value of the filtration parameter for different levels of noise as indicated at the top of each figure. At the top, results obtained from the graph and at the bottom, results obtained from the clique. The $95 \%$ confidence intervals for the mean of the normalized Betti numbers are of the size of the data points.

data. For these cases, however, the differences for $\delta=1 d$ become almost nonexistent. Indeed, as the levels of noise are augmented, distinguishing among the states requires larger values of $\delta$.

The results of the clique (bottom of Fig. 8) reveal that, opposite to the graph, the $\beta_{1}$ values are systematically smaller for the case of the highest tap intensity. Again, this reveals that states with the same packing fraction develop more triangles when obtained at high tap intensities. Concerning the differences among the states when adding noise and changing the filtration parameter, the conclusions attained from the clique are similar to those already explained for the graph. In summary, for low levels of noise, differences are maximized for intermediate values of $\delta$. As the noise is increased, the values of $\delta$ from which differences appear also increase. The curve trends (monotonously decreasing for the case without 
added noise, and displaying a maximum when some noise is added) can be explained, again, as a consequence of the development of triangles in the network.

\section{CONCLUSIONS}

In this work, we have shown that the first Betti number of both the graph and the clique (the Vietoris-Rips complex) can be satisfactorily used to classify granular packings. Using a filtration parameter that defines whether or not two particles in the sample (nodes) are joined by a link, we are able to differentiate among states that display the same packing fraction but which are, indeed, different.

We have studied the $\beta_{1}$ dependence on both $\Gamma$ and $\phi$ revealing the structural differences in particle arrangements where the global packing fraction are essentially the same. In general, increasing $\phi$ leads to an augment of the crystallization evidenced by an augment of the $\beta_{1}$ of the graph and a reduction of $\beta_{1}$ of the clique. The last is clearly due to the increasing number of triangles in the structure obtained when $\phi$ is augmented. In addition, we have found that the way in which the packing morphology changes with the packing fraction is not the same for high and low $\Gamma$. This is captured by the $\beta_{1}$ values for states with the same packing fraction which are, indeed, different for high and low $\Gamma$. Stationary states achieved using low values of $\Gamma$ are characterized by a smaller $\beta_{1}$ in the graph and larger in the clique, when compared with states with the same packing fraction but achieved using high values of $\Gamma$. This implies that the number of triangular structures is different among these two states: low values of $\Gamma$ lead to a smaller number of triangles than high values $\Gamma$. From this, we can infer that crystallization (measured by the number of triangular structures) is more important for high excitation intensities. This higher degree of crystallization should be compensated by the apparition of few big defects in order to share the same packing fractions as stationary states obtained for low excitation intensities.

The results reported in this work prove that an accurate determination of the contacts among the particles is not necessary to observe topological differences among states with the same packing fraction, but obtained with different tapping intensities. This result represents an important step forward with respect to a previous one [27] where the topological tool introduced to identify such differences is only available if the contact network is well defined. Clearly, the topological approach introduced in this work can be used to classify experimental packing ensembles where the contact network is not fully accessible due to the limited resolution of the experimental techniques.

\section{ACKNOWLEDGMENTS}

We would like to thank H. Adams and V. Nanda for fruitful discussions on the use of JAVAPLEX and PERSEUS respectively. R.A. thanks MIUR-FIRB RBFR081IUK for financial support. This work has been financially supported by Projects No. FIS2011-26675 and No. MTM2009-14409C02-01 (Spanish Government) and PIUNA (Universidad de Navarra). S.A. acknowledges the Institute for Mathematics and its Applications of the University of Minnesota where part of this research was completed.

\section{APPENDIX: SIMULATIONS}

We summarize the details of the simulations used in the analysis of the influence of indeterminacy in the positions of the particles. Further details can be found in [46]. We simulate $N=512$ monosized disks of diameter $d$, inside a two-dimensional confining box of width $13.39 d$ and infinitely high lateral walls. Note that the width of the simulated cell is roughly half of the cell's width in the experiments described in Sec. II. Hence, direct cuantitative comparison should not be done. Numerically, we set the stiffness $k_{n}=10^{5}(\mathrm{mg} / d)$ and damping parameter $\gamma_{n}=300(m \sqrt{g / d})$ in the normal direction of the contact. In the tangential direction, we set $k_{s}=\frac{2}{7} k_{n}$ for the stiffness and $\gamma_{s}=200(m \sqrt{g / d})$ for the damping parameter. The friction coefficient is fixed to $\mu=0.5$. We used reduced units with the diameter $d$ of the disks, the mass $m$, and the acceleration of gravity $g$. The integration time step is $\tau=10^{-4} \sqrt{d / g}$.

The tapping is simulated by moving the confining box in the vertical direction following a sine shaped trajectory $A \sin (2 \pi v t)[1-\Theta(2 \pi v t-\pi)]$. We fix the frequency at the value $v=\pi / 2(g / d)^{1 / 2}$ and control the tapping intensity $\Gamma=$ $A(2 \pi \nu)^{2} / g$ through the amplitude $A$. Once a tap is applied we decide that the system is in equilibrium implementing a criterium based on the stability of the contacts [46]. At this point, particle positions are recorded which will be subsequently used to calculate both the packing fraction and the network properties. Then, a new tap is applied. Following this protocol we tap the bed 1000 times for each reported value of the intensity. Averages are computed considering only the last 500 taps of each run, where (in all the cases) the packing fraction has already become stationary, i.e., it has a well defined average. The dependence of packing fractions against tapping amplitude is displayed in Fig. 9.

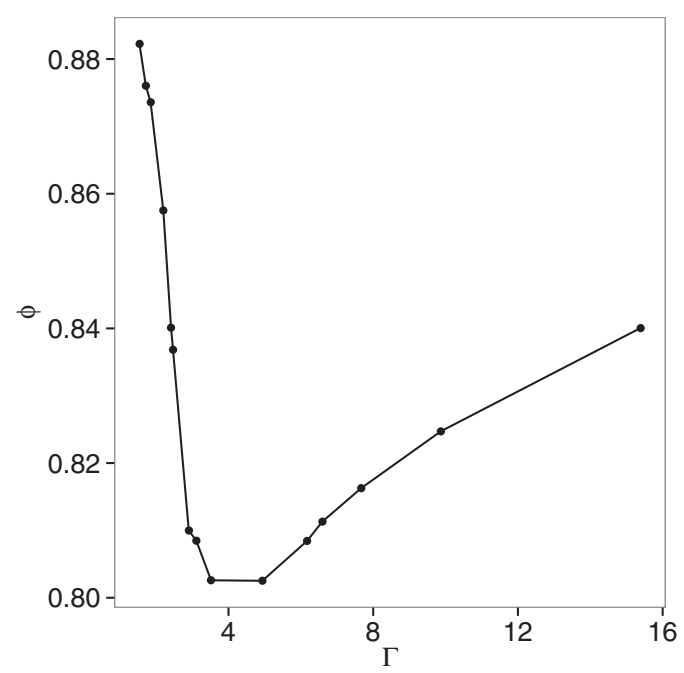

FIG. 9. Mean packing fraction $\phi$ of the steady states as a function of the tap intensity $\Gamma$ obtained from numerical simulations. 
[1] D. Bi, S. Henkes, K. E. Daniels, and B. Chakraborty, arXiv:1404.1854.

[2] S. F. Edwards and R. B. S. Oakeshott, Physica A 157, 1080 (1989).

[3] R. Blumenfeld and S. F. Edwards, J. Phys. Chem. B 113, 3981 (2009).

[4] R. Blumenfeld, J. F. Jordan, and S. F. Edwards, Phys. Rev. Lett. 109, 238001 (2012).

[5] M. E. J. Newman, SIAM Rev. 45, 167 (2003).

[6] P. M. Adler, Int. J. Multiphase Flow 11, 91 (1985).

[7] J. A. Dodds, J. Colloid. Interphase Sci. 77, 317 (1980).

[8] D. S. Bassett, E. T. Owens, K. E. Daniels, and M. A. Porter, Phys. Rev. E 86, 041306 (2012).

[9] D. M. Walker and A. Tordesillas, Phys. Rev. E 85, 011304 (2012).

[10] D. M. Walker and A. Tordesillas, Int. J. Solids Struct. 47, 624 (2010).

[11] D. M. Walker, A. Tordesillas, I. Einav, and M. Small, Phys. Rev. E 84, 021301 (2011).

[12] A. Tordesillas, D. M. Walker, E. Andò, and G. Viggiani, Proc. R. Soc. London, Ser. A 469, 2152 (2013).

[13] A. Tordesillas, D. M. Walker, and Q. Lin, Phys. Rev. E 81, 011302 (2010).

[14] A. G. Smart and J. M. Ottino, Phys. Rev. E 77, 041307 (2008).

[15] N. Rivier, J. Non-Cryst. Solids 352, 4505 (2006).

[16] N. S. Nguyen, H. Magoariec, B. Cambou, and A. Danescu, Int. J. Solids Struct. 46, 3257 (2009).

[17] N. S. Nguyen and H. Magoariec, B. Cambou. Int. J. Numer. Anal. Methods Geomech. 36, 1609 (2012).

[18] S. Ostojic, E. Somfai, and B. Nienhuis, Nature (London) 439, 828 (2006).

[19] R. Arévalo, I. Zuriguel, and D. Maza, Int. J. Bifurcation Chaos 19, 695 (2009).

[20] R. Arévalo, I. Zuriguel, and D. Maza, Phys. Rev. E 81, 041302 (2010).

[21] R. Arévalo, I. Zuriguel, S. Ardanza-Trevijano, and D. Maza, Int. J. Bifurcation Chaos 20, 897 (2010).

[22] D. M. Walker, A. Tordesillas, C. Thornton, R. P. Behringer, J. Zhang, and J. Peters, Granular Matter 13, 233 (2011).

[23] L. Kondic, A. Goullet, C. O’Hern, M. Kramar, K. Mischaikow, and R. Behringer, Europhys. Lett. 97, 54001 (2012).

[24] L. Kondic, X. Fang, W. Losert, C. S. O'Hern, and R. P. Behringer, Phys. Rev. E 85, 011305 (2012).
[25] G. Carlsson, J. Gorham, M. Kahle, and J. Mason, Phys. Rev. E 85, 011303 (2012).

[26] M. Oda and K. Iwasita (eds.), Mechanics of Granular Materials: An Introduction (A. A. Balkema, Roterdam, 1999).

[27] R. Arévalo, L. A. Pugnaloni, I. Zuriguel, and D. Maza, Phys. Rev. E 87, 022203 (2013).

[28] L. A. Pugnaloni, I. Sánchez, P. A. Gago, J. Damas, I. Zuriguel, and D. Maza, Phys. Rev. E 82, 050301R (2010).

[29] M. Kramar, A. Goullet, L. Kondic, and K. Mischaikow, Phys. Rev. E 87, 042207 (2013).

[30] J. Zhang, T. S. Majmudar, A. Tordesillas, and R. B. Behringer, Granular Matter 12, 159 (2010).

[31] A. Tordesillas, Q. Lin, J. Zhang, R. P. Behringer, and J. Shi, J Mech. Phys. Solids 59, 265 (2011).

[32] E. R. Nowak, J. B. Knight, E. Ben-Naim, H. M. Jaeger, and S. R. Nagel, Phys. Rev. E 57, 1971 (1998).

[33] E. R. Nowak, J. B. Knight, M. L. Povinelli, H. M. Jaeger, and S. R. Nagel, Powder Technol. 94, 79 (1997).

[34] J. A. Dijksman and M. van Hecke, Europhys. Lett. 88, 44001 (2009).

[35] P. Ribière, P. Richard, P. Philippe, D. Bideau, and R. Delannay, Eur. Phys. J. E 22, 249 (2007).

[36] M. Schröter, D. I. Goldman, and H. L. Swinney, Phys. Rev. E 71, 030301R (2005).

[37] M. Pica Ciamarra, A. Coniglio, and M. Nicodemi, Phys. Rev. Lett. 97, 158001 (2006).

[38] L. A. Pugnaloni, J. Damas, I. Zuriguel, and D. Maza, Pap. Phys. 3, 030004 (2011).

[39] G. Carlsson, Bull. Am. Math. Soc. 46, 255 (2012).

[40] H. Edelsbrunner and J. L. Harer, Computational Topology (AMS, Providence, RI, 2010).

[41] A. J. Zomorodian, Topology for Computing (Cambridge University Press, Cambridge, UK, 2005).

[42] A. Tausz, M. Vejdemo-Johansson, and H. Adams, JavaPlex: A Research Software Package for Persistent (co)Homology (2011) http://code.google.com/javaplex.

[43] K. Mischaikov and V. Nanda, Discrete Comput. Geom. 50, 330 (2013).

[44] Perseus: The Persistent Homology Software. http://www.sas.upenn.edu/vnanda/perseus. Accessed 14/02/14.

[45] S. Slotterback, M. Mailman, K. Ronaszegi, M. van Hecke, M. Girvan, and W. Losert, Phys. Rev. E 85, 021309 (2012).

[46] R. Arévalo, D. Maza, and L. A. Pugnaloni, Phys. Rev. E 74, 021303 (2006). 